\title{
Identificação do Perfil da Ionosfera Utilizando Lógica Nebulosa: Parte I
}

\author{
V.G. PILLAT ${ }^{*}$ e L.N.F. GUIMARÃES ${ }^{2}$ \\ Recebido em 20 novembro, 2013 / Aceito em 9 abril, 2014
}

\begin{abstract}
RESUMO. A ionossonda digital é um dos instrumentos que mede a densidade eletrônica da baixa ionosfera, através de rádio frequência, obtendo como resultado espectros de frequência em função da altura chamados ionogramas. A ionosfera pode ser analisada estudando-se alguns parâmetros críticos que indicam a altura da base da camada $F(h$ 'F), a frequência com o pico de densidade eletrônica na camada $F$ (foF2) e a altura do pico de densidade eletrônica na camada $\mathrm{F}(\mathrm{hpF} 2)$. Até o presente momento, estes parâmetros são extraídos dos ionogramas de uma forma manual e dependente da interpretação de um analista. Devido à grande quantidade de dados coletados pelas ionossondas necessita-se de uma automatização deste processo de coleta e análise. Este trabalho propõe um modelo de apoio à decisão para o processo de análise dos dados coletados por ionossondas com o propósito de elevar a capacidade de compilação dos dados ionosféricos. A implementação do modelo de apoio à decisão foi estruturada em duas partes: a primeira parte propõe uma abordagem de lógica nebulosa para o problema de associação de dados, considerando as diferentes camadas ionosféricas. A segunda parte propõe o emprego de relação nebulosa como estrutura para a construção das regras de um sistema nebuloso especialista. Este trabalho apresenta apenas a primeira parte do modelo.
\end{abstract}

Palavras-chave: lógica nebulosa, relação nebulosa, ionograma, ionosfera.

\section{INTRODUÇÃO}

A atmosfera terrestre é formada por átomos e moléculas neutras, átomos e moléculas ionizados e elétrons livres. De acordo com o perfil vertical de temperatura a atmosfera neutra pode ser subdividida em troposfera [0-15 km], estratosfera [15-50 km], mesosfera [50-80 km] e termosfera [80-650 km]. De acordo com a densidade eletrônica a atmosfera ionizada (ionosfera) é subdividida em camada D [70-90 km], camada E [90-150 km] e camada F [150-1000 km]) [2].

O estudo da ionosfera tornou-se ainda mais relevante na sociedade moderna devido a grande dependência que as novas tecnologias tem em relação aos satélites. Além, dos satélites orbitarem

\footnotetext{
*Autor correspondente: Valdir Gil Pillat

${ }^{1}$ Laboratório de Física e Astronomia, UNIVAP - Universidade do Vale do Paraíba, 12244-000, São José dos Campos, SP, Brasil / Programa de Doutorado em Computação Aplicada, INPE - Instituto Nacional de Pesquisas Espaciais, 12227-010 São José dos Campos, SP, Brasil. E-mail: valdirgp@univap.br

${ }^{2}$ Divisão de Energia Nuclear, IEAv - Instituto de Estudos Avançados, 12228-970 São José dos Campos, SP, Brasil. E-mail: guimarae@ieav.cta.br
} 
principalmente em altitudes ionosféricas as transmissões satélite-satélite e satélite-estação terrestre são realizadas por intermédio de ondas eletromagnéticas, e essas ondas interagem (refração, reflexão e absorção) com a atmosfera ionizada quando se propaga.

O estudo dessa região da atmosfera pode ser realizado utilizando equipamentos em solo, tais como: ionossondas, radares de espalhamento coerente e incoerente, GPS e equipamentos ópticos. Um dos equipamentos mais utilizados para estudar a ionosfera é um sistema de radar chamado ionossonda. As ionossondas operam na faixa de rádio frequências de 1 a 20-30 MHz. Os sinais de rádio frequência transmitidos pela ionossonda são refletidos quando a frequência transmitida for igual à frequência natural do plasma ionosférico, ou seja, um eco retorna para a superfície da Terra. Medindo tempo decorrido entre a transmissão e a recepção do sinal e assumindo-se que a onda se propaga com a velocidade da luz no vácuo calcula-se a altura virtual onde ocorreu à reflexão.

O gráfico resultante desses ecos, quando se realiza uma varredura de frequências é chamado de ionograma. Esse gráfico fornece um traço característico de frequência versus a altura virtual (Figura 1). Nesse traço é possível identificar as camadas E, F1 e F2.

Atualmente, com o advento das ionossondas digitais é possível obter ionogramas com uma resolução temporal significativa, entre 1 a 5 minutos. Portanto, a utilização de técnicas computacionais para visualizar, filtrar ruídos e extrair parâmetros ionosféricos do traço característico dos ionogramas, tornou-se uma ferramenta indispensável nessa área de pesquisa. Na realidade, a extração desses parâmetros automaticamente é importante para manipularmos uma grande quantidade de ionogramas.

Existem diversos fabricantes de ionossonda, como por exemplo, Digissonda ${ }^{\mathrm{TM}}$ fabricada pela "University of Massachusetts Lowell's Center for Atmospheric Research" (UMLCAR), "Canadian Advanced Digital Ionosonde" (CADI) fabricada pela Canadian Network for Space Research e "Advanced Ionospheric Sounder - Istituto Nazionale di Geofisica e Vulcanologia" (AIS-INGV) fabricada pelo "Istituto Nazionali di Geofisica e Vulcanologia" (INGV). Porém, o programa para extrair os parâmetros ionosféricos são fabricados especificamente para cada modelo de ionossonda, para a Digissonda existe o ARTIST, para a AIS-INGV existe o Autoscala, e para a CADI não existe uma ferramenta para realizar esta tarefa. O objetivo deste trabalho é desenvolver uma ferramenta para extração dos parâmetros ionosféricos para a ionossonda CADI.

As ionossondas utilizadas nesse trabalho pertencem ao Laboratório de Física e Astronomia da "Universidade do Vale do Paraíba" (UNIVAP). Atualmente o laboratório opera três ionossondas digitais, sendo que as ionossondas foram estrategicamente posicionadas quase alinhadas ao longo do meridiano magnético: São José dos Campos (SP), Palmas (TO) e Manaus (AM). Neste trabalho são utilizados apenas os dados da ionossonda de São José dos Campos $\left(23.2^{\circ} \mathrm{S}, 45.9^{\circ} \mathrm{W}\right.$; dip latitude $\left.17.6^{\circ} \mathrm{S}\right)$.

A CADI possibilita realizar observações em dois modos diferentes: o primeiro modo varre 180 frequências no intervalo de 1 a $20 \mathrm{MHz}$, sendo que a coleta destes dados ocorre em um intervalo de 300 segundos. O arquivo gerado desta forma é chamado MD4 [4]. Em 30 dias são obtidos 
8640 ionogramas completos; e o segundo modo varre apenas 6 frequências diferentes, a saber: 3, 4, 5, 6, 7, $8 \mathrm{MHz}$. Sendo que a coleta destes dados ocorre em um intervalo de tempo de 100 segundos. O arquivo gerado desta forma é chamado MD3 [4]. A extração dos parâmetros ionosféricos é realizada sobre os ionogramas completos.

Para realizar uma análise da ionosfera é preciso analisar um conjunto de ionogramas. A dificuldade desta análise está no fato que são necessários vários ionogramas e os ionogramas contêm um número grande de pontos. O estudo da dinâmica da ionosfera é realizado através da extração de alguns parâmetros ionosféricos que contêm a física do fenômeno. Devido à dificuldade de extração dos parâmetros ionosféricos o Laboratório de Física e Astronomia da UNIVAP desenvolveu um programa que torna mais simples a manipulação dos dados dos ionogramas e a extração dos parâmetros ionosféricos. Esta ferramenta computacional foi denominada "Univap Digital Ionosonde Data Analysis" (UDIDA) [5, 7]. A Figura 1 ilustra a visualização de um ionograma utilizando o UDIDA.

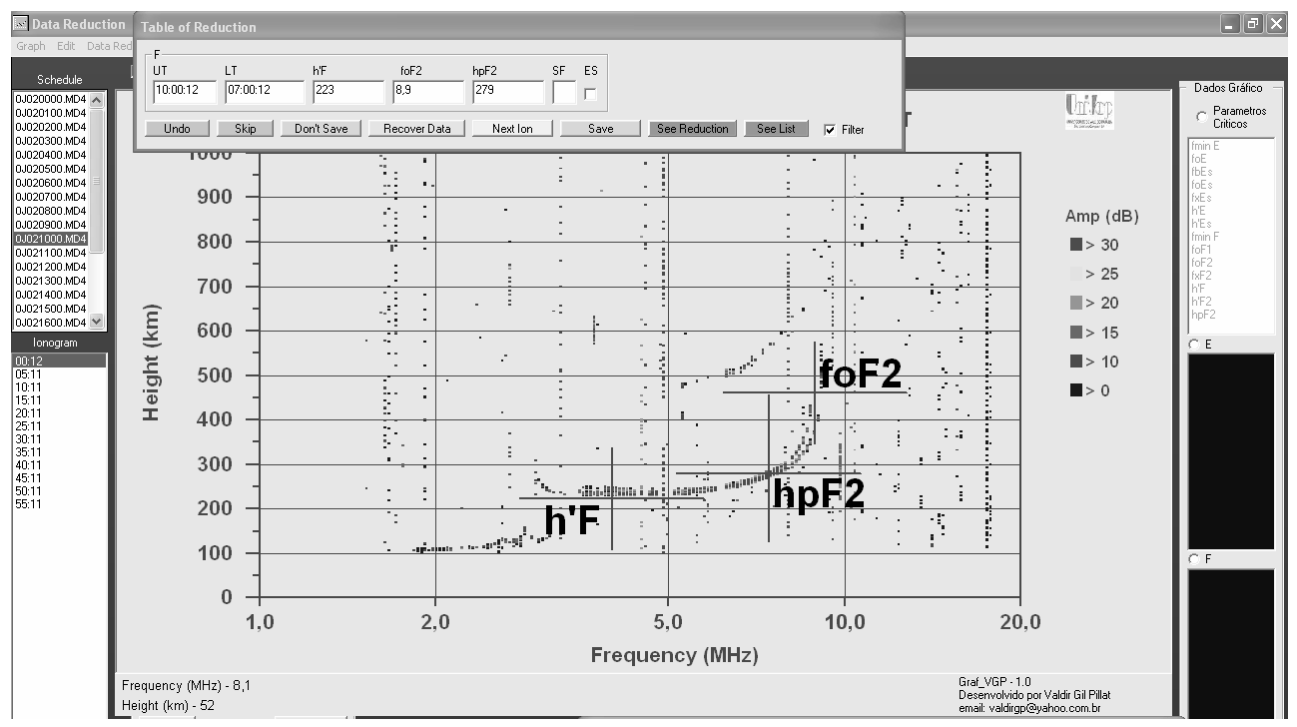

Figura 1: Exemplo de um ionograma obtido pela ionossonda digital do tipo CADI em 02 de outubro de 2000, São José dos Campos, visualizado e extraído manualmente os parâmetros ionosféricos, h'F, hpF2 e foF2, utilizando o programa UDIDA.

Estes parâmetros ionosféricos são: altura virtual da base da camada $F$ (h'F), a frequência com o pico de densidade eletrônica na camada $\mathrm{F}$ (foF2) e altura virtual do pico de densidade eletrônica na camada $\mathrm{F}$ (hpF2). Estes parâmetros são importantes no estudo da dinâmica da ionosfera sob várias condições geomagnéticas, pois a variação temporal deles fornece este tipo de informação. Observe que, no caso do UDIDA, a extração dos parâmetros ionosféricos é manual e dependente da interpretação do analista, o que pode gerar erros de interpretação e julgamento. Visando uma padronização neste processo surgiu a ideia de implementar paradigmas baseados em Inteligência Computacional (IC) para automatizar a extração dos parâmetros ionosféricos, dos ionogramas 
gerados pela ionossonda CADI. Desta forma, a sistematização do processo de análise de dados tem o propósito de elevar a capacidade de extração dos parâmetros ionosféricos a partir dos ionogramas.

\section{METODOLOGIA}

Observando que muitos conceitos no mundo real não podem ser bem representados usando limites claramente definidos, Zadeh (1965) desenvolveu a teoria dos conjuntos nebulosos (Fuzzy Sets) para tratar do aspecto vago da informação [12]. O fundamento matemático dessa teoria pode ser tratado como uma generalização da teoria dos conjuntos clássica. Essa teoria generaliza os conceitos da teoria clássica de conjuntos para o tratamento da imprecisão da informação na área de controle e na área de decisão, buscando traduzir em termos formais a informação imprecisa que ocorre de maneira natural na representação dos fenômenos da natureza, descritos por seres humanos.

As informações coletadas pelas ionossondas podem estar contidas em dois tipos de dados: dados numéricos recebidos das medições do radar e dados linguísticos obtidos de analistas, pesquisadores e outras fontes. Em situações do mundo real, os dados numéricos podem ser ruidosos, inconsistentes e incertos e as informações linguíticas podem ser imprecisas ou vagas. Em um sistema como a ionossonda a incerteza pode ser gerada por outros sinais transmitidos na faixa de frequência medida, radio amador, ou por falha/inacurácia no equipamento na coleta dos dados.

As variáveis linguísticas são expressões que caracterizam grandezas físicas e cujo valor é especificado por proposições da linguagem natural em detrimento de um valor numérico [8].

Este trabalho aborda um modelo de apoio à decisão para o processo de extração de parâmetros ionosféricos a partir de ionogramas. Este modelo foi estruturado em duas partes. A primeira parte propõe uma abordagem de lógica nebulosa para o problema de associação de dados, classificando os dados conforme a camada da ionosfera a que ele pertence. Nesta parte são aprimoradas as fases de observação e de orientação do modelo pela otimização do processamento de dados de modo que o modelo processe de forma isolada cada camada ionosférica. Nesta etapa é utilizada a relação nebulosa. A segunda parte propõe a construção de regras nebulosas pelos resultados obtidos na relação nebulosa. A lógica nebulosa foi escolhida devido a capacidade de agregar as heurísticas empregadas pelos analistas. Neste trabalho é apresentada a primeira parte deste modelo.

No ionograma ilustrado na Figura 2 é possível observar as interferências junto com a reflexão da ionosfera, regiões marcadas com três elipses. Estas interferências em determinados casos dificultam a identificação dos parâmetros ionosféricos. As interferências são as linhas verticais que identificam uma frequência relacionada a algum efeito externo, como por exemplo, a frequência transmitida por rádio amador.

Para que seja possível a implementação da extração automática dos parâmetros ionosféricos é necessário analisar os dados obtidos pela ionossonda e definir os passos que são realizados manualmente. Após este processo foram identificados alguns passos intermediários antes da extração dos parâmetros de maneira automática. Basicamente, são necessários os seguintes passos: 


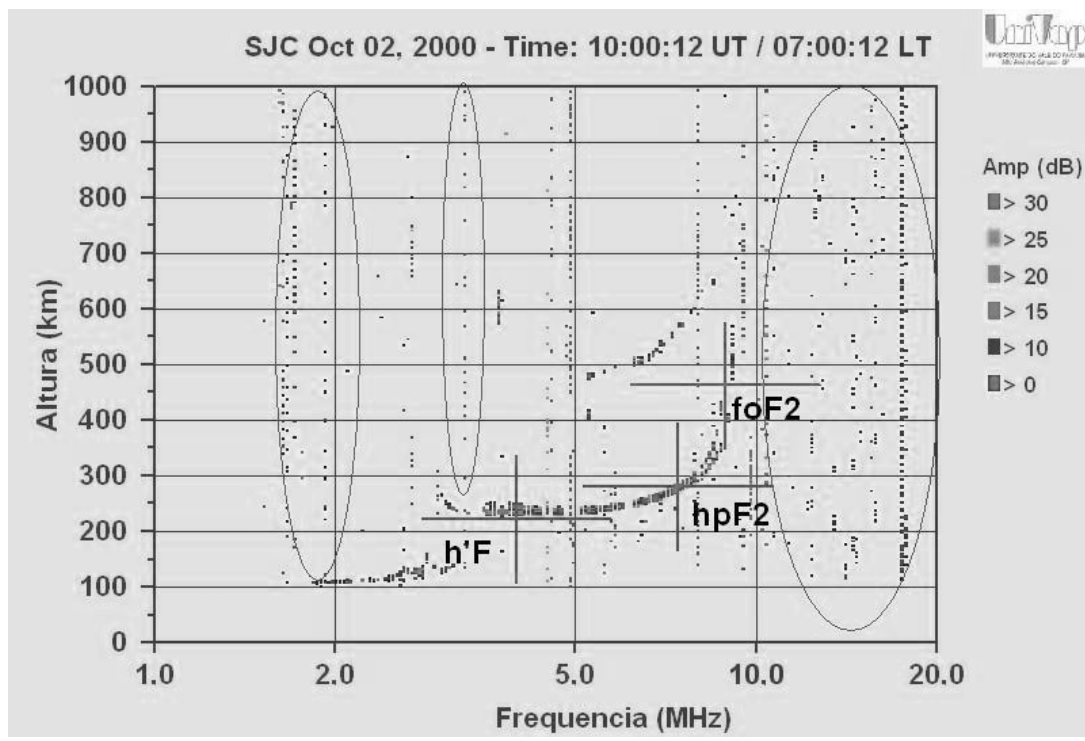

Figura 2: Exemplo de ionograma obtido em São José dos Campos em 02 de outubro de 2000, com as interferências e os parâmetros ionosféricos indicados.

- Tratar o ionograma como uma figura.

- Filtragem das interferências: os dados obtidos pela ionossonda vêm com interferências (Figura 2) que podem ser provocadas, por exemplo, por rádio amador. Estas interferências podem complicar a identificação dos parâmetros ionosféricos. A técnica implementada nesta etapa de remoção de interferências, trata o ionograma como uma imagem e o divide em sub-regiões que contêm uma quantidade de pontos. Se esta sub-região contém menos de 5 pontos é considerada interferência e descartada, permitindo uma melhor visualização do perfil da ionosfera [6].

- Definir a região onde está contido o perfil da ionosfera. A complexidade desta etapa está na dinâmica da ionosfera onde sua densidade eletrônica é alterada devido a diversos fenômenos físicos/químicos resultando em diversas formas do perfil inferior da ionosfera. Utilizando relação nebulosa é possível determinar esta região, conforme é apresentado a seguir.

- Extração dos parâmetros ionosféricos. A lógica nebulosa pode ser aplicada a esta etapa conforme trabalhos anteriores, $[10,1]$ onde através da defuzificação é possível extrair um valor de uma região.

Em abordagem nebulosa, as relações possuem a potência computacional e a importância que as funções possuem em abordagens convencionais. Relações nebulosas podem ser comparadas com os conjuntos nebulosos definidos sobre um universo de discurso. Como o nome indica, uma relação implica na presença de uma associação entre elementos de diferentes conjuntos. 
Neste trabalho, utiliza-se a relação nebulosa na identificação do perfil da ionosfera. A maneira como a lógica nebulosa é aplicada neste trabalho é diferente, visto que a lógica nebulosa é bem difundida na área de controle. A Figura 3 ilustra um exemplo de como seria a região onde o perfil da ionosfera estaria contido (linha em preto) definida pela relação nebulosa $[3,9,11]$. A definição desta região é importante para diminuir a área de atuação do algoritmo de extração dos parâmetros.

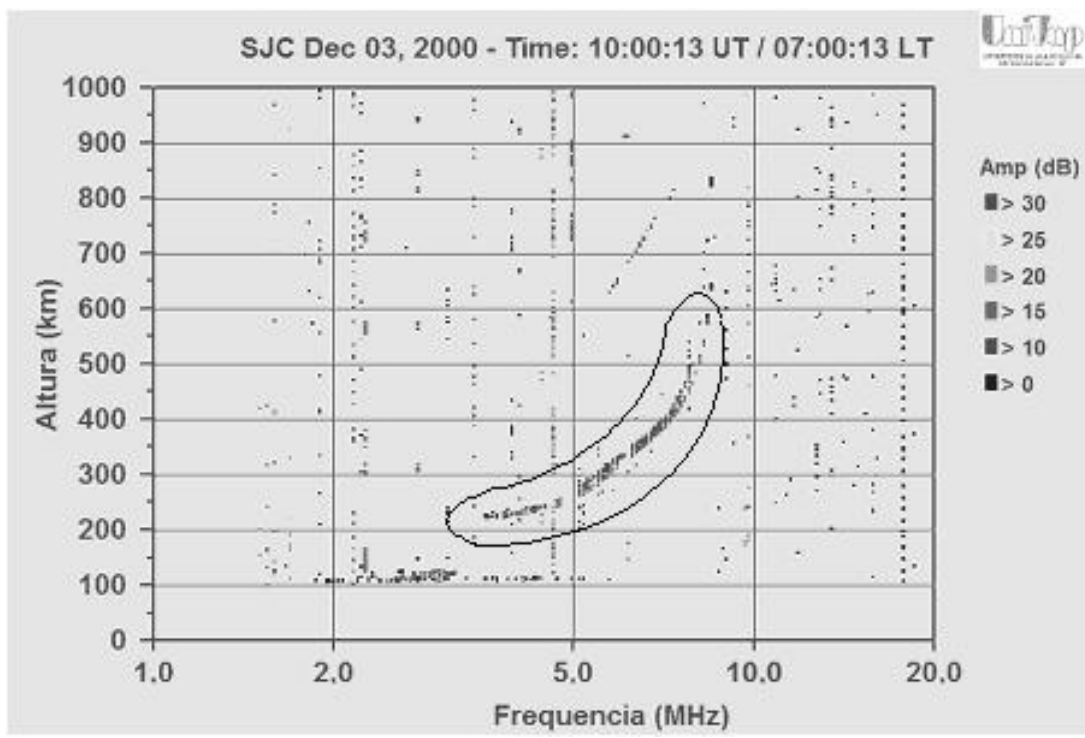

Figura 3: Ionograma com a região onde está presente o perfil da ionosfera identificado utilizando a relação nebulosa.

Como mencionado, a ionosfera pode ser classificada em camadas concêntricas da superfície da Terra de acordo com seus níveis de densidade eletrônica, (D [70 a 90 km], E [90 a 150 km] e F [150 a $1000 \mathrm{~km}]$ ). A ionossonda CADI desconsidera os ecos abaixo de 100km de altitude, portanto nos ionogramas obtidos por esta ionossonda é possível visualizar as camadas $\mathrm{E}$ e F da ionosfera. Propõe-se que a classificação dos dados do ionograma seja realizada conforme os seguintes passos:

- Definir o conjunto nebuloso para as frequências do ionograma, conforme as funções de pertinência ilustradas na Figura 4.

Na Figura 4 é ilustrada a função de pertinência triangular utilizada para definir os valores de pertencimento das frequências medidas. A função triangular foi utilizada por ser de fácil interpretação numérica. Cada triângulo representa uma camada ionosférica, a saber: E (1 a 3), Es (1 a 6), F1 (1 a 6), F2 (3,0 a 10) e F3 (5 a 18). A definição dos limites de cada triângulo foi realizada via experimentação numérica e utilizando a experiência dos analistas na área de ionosfera. Uma forma de representar este conjunto nebuloso linguisticamente é: A frequência 


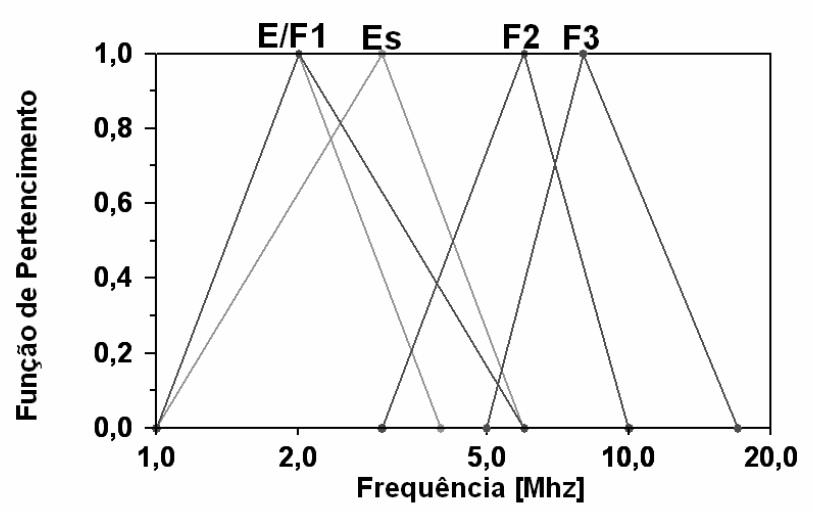

Figura 4: Conjunto nebuloso para as frequências do ionograma. A frequência está representada em escala logarítmica.

f pertence à camada $\mathrm{X}$ com grau de pertinência Y. Por exemplo: A frequência 5,0 pertence à camada Es com grau de pertinência 0,3 , pertence à camada $\mathrm{F} 1$ com grau de pertinência $0,2 \mathrm{e}$ pertence a camada F2 com grau de pertinência 0,65 .

- Definir o conjunto nebuloso para as altitudes, conforme as funções de pertinência ilustradas na Figura 5.

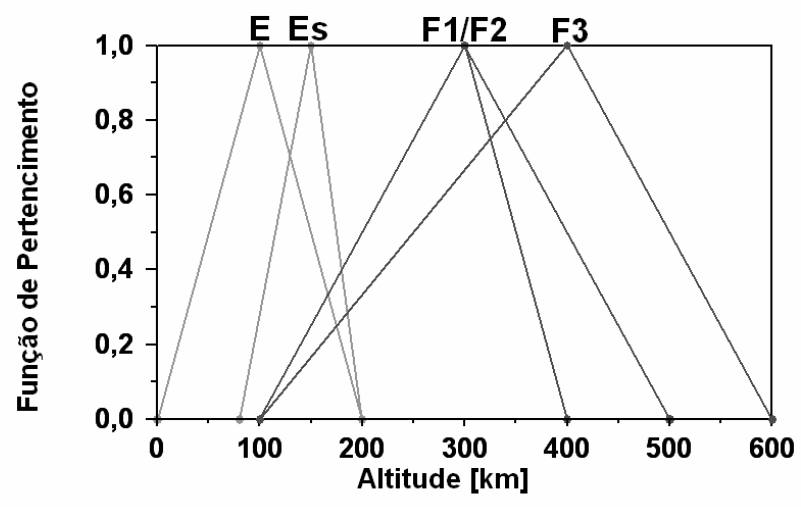

Figura 5: Conjunto nebuloso para as altitudes do ionograma.

Na Figura 5 é ilustrada a função de pertinência triangular utilizada para definir os valores de pertencimento das altitudes medidas. Cada triângulo representa uma camada ionosférica, a saber: E (0 a 200), Es (80 a 200), F1 (150 a 400), F2 (150 a 500) e F3 (150 a 600). Uma forma de representar este conjunto nebuloso linguisticamente é: A altitude h pertence à camada $\mathrm{X}$ com 
grau de pertinência Y. Por exemplo: A altitude 180 pertence à camada E com grau de pertinência 0,20 , pertence à camada Es com grau de pertinência 0,60 , pertence à camada $\mathrm{F} 1$ com grau de pertinência 0,35 , pertence à camada $\mathrm{F} 2$ com grau de pertinência 0,35 e pertence à camada $\mathrm{F} 3$ com grau de pertinência 0,2 .

- Produzir a relação entre os dois conjuntos, é definido uma relação nebulosa que se adeque melhor aos dados. A relação nebulosa é definida quando um ponto pertence a uma região se este apresentar um valor de pertinência maior ou igual a 0,5 nos dois conjuntos (frequência e altitude). O resultado desta relação são retângulos para cada região que são ilustrados na Figura 6 nos resultados.

\section{RESULTADOS}

Foram realizados alguns testes da aplicação deste método de classificação nos ionogramas e foi constatado que para um dia calmo o método apresenta bons resultados, visto que na maioria dos ionogramas o perfil da ionosfera está contido na área definida pela relação nebulosa. Observe que é necessário definir uma distribuição diferente dos limites dos triângulos, conforme o período do dia. Assim gerando dois conjuntos nebulosos diferentes referente a cada período, um no período das $8 \mathrm{~h}-17 \mathrm{~h}$ tempo local (presença do Sol) e outro das 18h-7h tempo local (ausência do Sol). Na Figura 6 são mostradas algumas classificações realizadas no dia 01 de outubro de 2000 na estação de São José dos Campos.
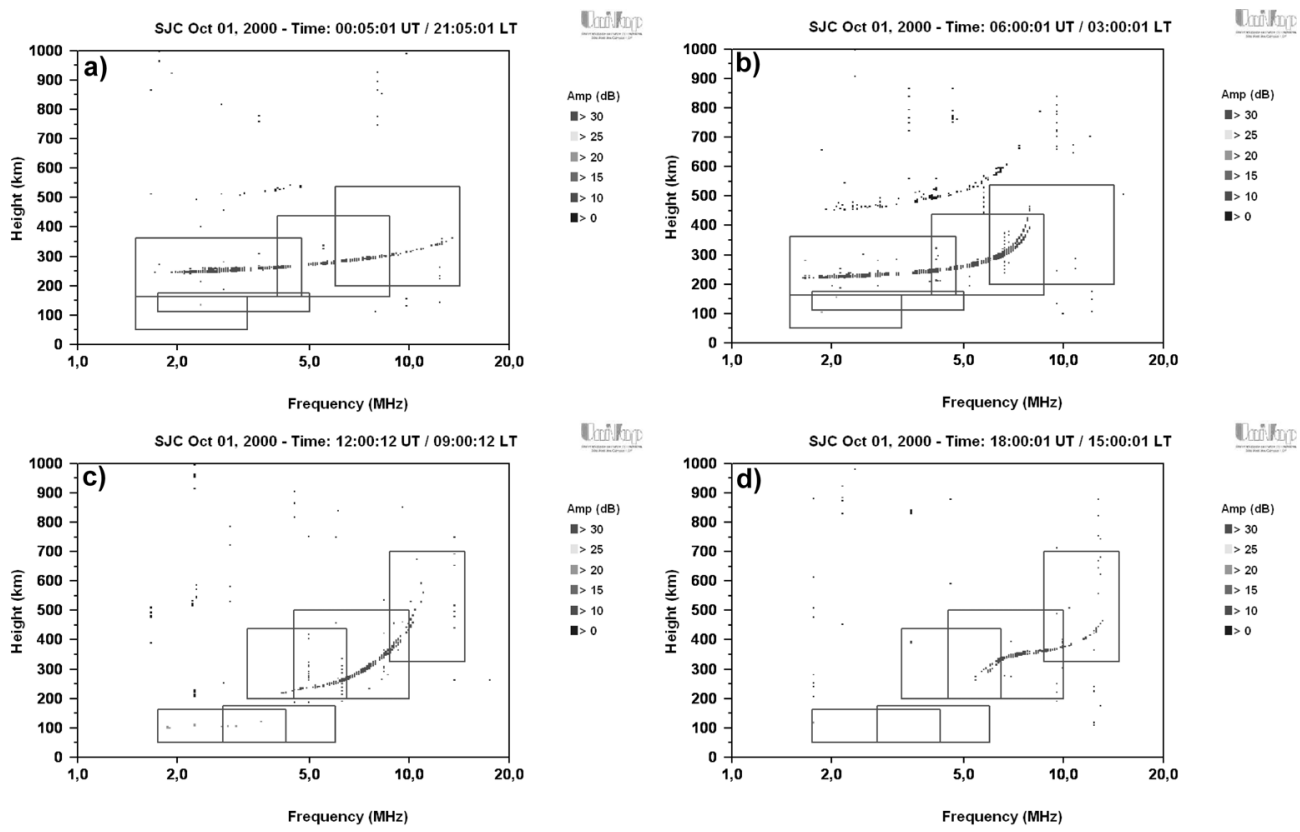

Figura 6: Exemplos da classificação dos dados do ionograma utilizando a relação nebulosa nos ionogramas obtidos em 01 de outubro de 2000. 
Na Figura 6 os retângulos indicam os limites da camada referente. Existem 5 retângulos no ionograma referente a cada camada da ionosfera que são: E, Es, F1, F2 e F3. A camada F foi dividida em 3 partes para colocar versatilidade na curva da relação.

As figuras 6 "a" e "b" são ionogramas obtidos na ausência do Sol e as figuras 6 "c" e "d" são ionogramas obtidos com a presença do Sol, observe que os retângulos contornam o perfil da ionosfera por completo. Note que, a distribuição dos retângulos para as figuras 6 "a" e "b" são diferentes dos retângulos das figuras "c" e "d". Os conjuntos nebulosos mostrados nas figuras 4 e 5 foram aplicados também para os ionogramas obtidos em 23 de junho de 2003 e 01 de janeiro de 2004 e os resultados não foram bons. Em alguns horários a relação não cobria o perfil da ionosfera, pois ficava fora da região definida no conjunto nebuloso, conforme é mostrado na Figura 7.

As figuras 7 "a" e "b" são ionogramas obtidos em 23 de junho de 2003, observe que na figura 7a o perfil da ionosfera possui uma densidade eletrônica baixa comparada com a definida nos conjuntos nebulosos. Na Figura $7 \mathrm{~b}$ o perfil da camada F inicia antes da frequência indicada nos conjuntos nebulosos.
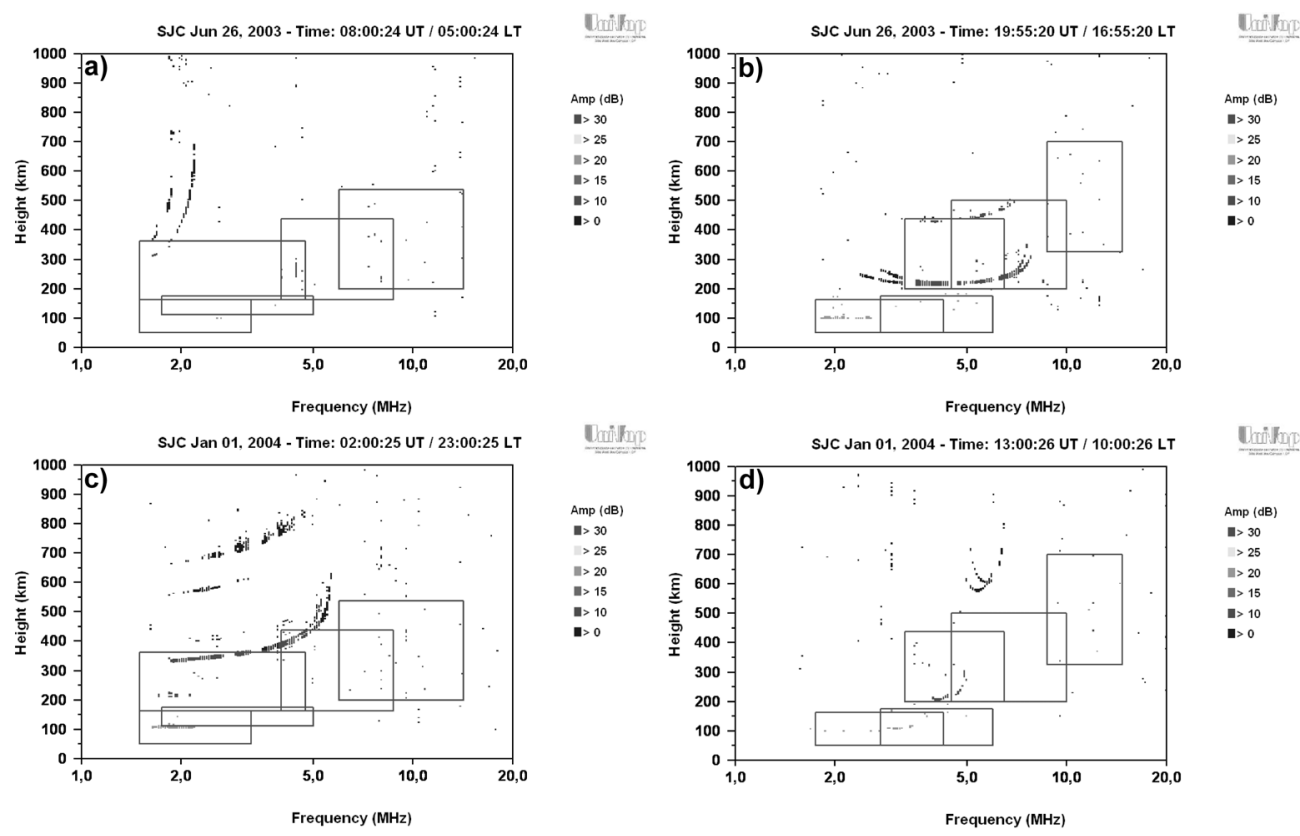

Figura 7: Exemplos da aplicação da relação nebulosa nos ionogramas obtidos em 23 de junho de 2003 e 01 de janeiro de 2004.

As figuras 7 "c" e "d" são ionogramas obtidos em 01 de Janeiro de 2004, observe que na Figura 7c o perfil da camada F é parcialmente contornado pelos retângulos e na Figura 7d o perfil da camada F está dividido em duas partes e os retângulos contornam apenas uma pequena região. 
Esse problema ocorre, pois a ionosfera tem um comportamento dinâmico e depende muito da atividade solar. Uma solução para este caso é recalcular os limites das camadas ionosféricas nos conjuntos nebulosos conforme a distribuição dos pontos no ionograma, ou seja, verificar onde termina a reflexão da ionosfera para cada ionograma e recalcular os limites de cada camada na função de pertencimento. Aplicar uma heurística adaptativa onde a heurística dos analistas é adaptada a cada ionograma carregado. Este é o próximo passo deste desenvolvimento.

\title{
4 CONCLUSÃO
}

Neste trabalho foi possível observar que a relação nebulosa é uma ferramenta poderosa no processo de classificação do ionograma. Este modelo ainda precisa de alguns ajustes para se adequar às variações na dinâmica da ionosfera. Algumas atividades futuras são apresentadas a seguir:

- Testar novos conjuntos nebulosos, através da variação das regiões de frequência e altitude, para verificar qual o melhor modelo para determinar os limites das camadas ionosféricas;

- Definir as regras nebulosas para extração dos parâmetros ionosféricos;

- Testar diferentes lógicas (Mamdani, Larsen e Takagi-Sugeno) verificando o processo de defuzificação; e

- Comparar os parâmetros ionosféricos extraídos pelo modelo com os parâmetros extraídos manualmente por analistas.

\begin{abstract}
The ionosonde is a digital instrument that measures the electron density of the lower ionosphere by radio frequency, obtaining a result a frequency spectra as a function of time called ionograms. The ionosphere can be analyzed by studying a few critical parameters that indicate the height of the base layer F (h'F), the peak electron density frequency in the $\mathrm{F}$ layer (foF2) and the height of peak electron density in the layer $\mathrm{F}$ (hpF2). To date, these parameters are extracted from ionograms in a manual and on the analyst interpretation. Due to the large amount of data collected by the ionosonde there is a need of automation of this process of collection and analysis. This paper proposes a model of decision support for the process of analyzing data collected by ionosonde for the purpose of raising the capacity building of ionospheric data. The implementation of decision support model was structured in two parts: the first part proposes a fuzzy logic approach to the problem of data binding, considering the different ionospheric layers. The second part proposes the use of fuzzy relation as a framework for the construction of the rules of a fuzzy expert system. This work addresses the first part of the model.
\end{abstract}

Keywords: fuzzy logic, fuzzy relation, ionogram, ionosphere.

\section{REFERÊNCIAS}

[1] F.T. Berkey \& P. Sikdar. Studies of the temperate e-layer using a windowed fuzzy clustering technique. Proceedings of the 11th Ionospheric Effects Symposium, Alexandria, VA, 6B, n. Issue 3, p. A082, (2005). 
[2] J.A. Bittencourt. "The Low Latitude Ionosphere: A Dynamic Computer Model", São José dos Campos: INPE, [INPE-5965-RPQ/674], p. 214, (1996).

[3] G.J. Klir. "Fuzzy Sets and Fuzzy Logic: Theory and Applications". Upper Saddle River, p. 563, (1995).

[4] J.W. Macdougall, I.F. Grant \& X. Shen. "The Canadian advanced digital ionosonde: design and results”. URSI INAG Ionospheric Station Inf. Bulletin, UAG-104, (1995).

[5] V.G. Pillat. "Estudo da ionosfera em baixas através do modelo computacional LION e comparação com parâmetros ionosféricos observados". 160 p. Dissertação (Mestrado em Física e Astronomia), UNIVAP, São José dos Campos, (2006).

[6] V.G. Pillat, L.N.F. Guimarães, J.D.S. Silva \& P.R. Fagundes. Filtragem de dados de radar visando a aplicação de lógica nebulosa. WORCAP, 8, INPE, São José dos Campos, SP, (2008).

[7] V.G. Pillat \& P.R. Fagundes. UDIDA UNIVAP DIGITAL IONOSONDE DATA ANALYSIS. VII Encontro Latino Americano de Iniciação Científica e IV Encontro Americano de Pós-Graduação - EPG, São José dos Campos, 4 (2004), 1178-1184.

[8] S. Sandri \& C. Correa. "Lógica nebulosa". Escola de redes neurais: Conselho Nacional de Redes Neurais, ITA São José dos Campos, 5 (1999), 73-90.

[9] K. Tanaka. "Introduction to Fuzzy Logic Pratical applications", New York, (1997), 148 p.

[10] L.-C. Tsai \& F. Berkey. Ionogram analysis using fuzzy segmentation and connectedness techniques. Radio Sci., 35 (2000), 1173-1186.

[11] L.H. Tsoukalas \& R.E. Uhrig. "Fuzzy and Neural Approaches in Engineering", Nova York, (1997), $600 \mathrm{p}$.

[12] L.A. Zadeh. "Fuzzy sets". Information and Control, 8 (1965), 338-353. 